\title{
Benchmark of a multi-physics Monte Carlo simulation of an ion guide for neutron-induced fission products
}

\author{
Z. Gao ${ }^{1, a}$, A. Al-Adili ${ }^{1}$, L. Cañete ${ }^{2}$, T. Eronen ${ }^{2}$, D. Gorelov ${ }^{2}$, A. Kankainen ${ }^{2}$, M. Lantz ${ }^{1}$, A. Mattera ${ }^{3}$, I. D. Moore ${ }^{2}$, \\ D. A. Nesterenko ${ }^{2}$, H. Penttilä ${ }^{2}$, I. Pohjalainen ${ }^{2}$, S. Pomp ${ }^{1}$, V. Rakopoulos ${ }^{1}$, S. Rinta-Antila ${ }^{2}$, M. Vilén ${ }^{2}$, J. Äystö ${ }^{2}$, \\ A. Solders ${ }^{1, \mathrm{~b}}$ \\ ${ }^{1}$ Department of Physics and Astronomy, Uppsala University, BOX 516, 75120 Uppsala, Sweden \\ 2 Department of Physics, University of Jyväskylä, 40014 Jyväskylä, Finland \\ ${ }^{3}$ National Nuclear Data Center, Brookhaven National Laboratory, Building 817, Upton, NY 11973-5000, USA
}

Received: 23 August 2021 / Accepted: 29 January 2022 / Published online: 12 February 2022

(C) The Author(s) 2022

Communicated by Navin Alahari

\begin{abstract}
To enhance the production of medium-heavy, neutron-rich nuclei, and to facilitate measurements of independent yields of neutron-induced fission, a proton-toneutron converter and a dedicated ion guide for neutroninduced fission have been developed for the IGISOL facility at the University of Jyväskylä. The ion guide holds the fissionable targets, and the fission products emerging from the targets are collected in helium gas and transported to the downstream experimental stations. A computer model, based on a combination of MCNPX for modeling the neutron production, the fission code GEF, and GEANT4 for the transport of the fission products, was developed. The model will be used to improve the setup with respect to the production and collection of fission products. In this paper we benchmark the model by comparing simulations to a measurement in which fission products were implanted in foils located at different positions in the ion guide. In addition, the products from neutron activation in the titanium foil and the uranium targets are studied. The result suggests that the neutron flux at the high-energy part of the neutron spectrum is overestimated by approximately $40 \%$. However, the transportation of fission products in the uranium targets agrees with the experiment within $10 \%$. Furthermore, the simulated transportation of fission products in the helium gas achieves almost perfect agreement with the measurement. Hence, we conclude that the model, after correction for the neutron flux, is well suited for optimization studies of future ion guide designs.
\end{abstract}

\footnotetext{
${ }^{a}$ e-mail: zhihao.gao@physics.uu.se (corresponding author)

b e-mail: andreas.solders@physics.uu.se
}

\section{Introduction}

At the University of Jyväskylä, the Ion Guide Isotope Separator On-Line (IGISOL) technique is used to produce radioactive ion beams of short-lived exotic nuclei for fundamental nuclear physics research [1]. The main production mechanism for neutron-rich medium-heavy nuclei is proton- or deuteron-induced fission in actinide targets, such as uranium and thorium. However, it is anticipated that neutron-induced fission would provide access to even more exotic isotopes [2].

The IGISOL technique is also used to study the fission process itself through measurements of independent and isomeric fission yields (FY) [3-5]. Accurate fission yield data as a function of target material and excitation energy, as well as inducing particle type, are needed to achieve a better understanding of the fission process. Furthermore, yield distributions from neutron-induced fission at incident energies above thermal neutron energies are required in the design and development of Generation IV fast reactors.

In order to access neutron-rich nuclides further from stability, and to facilitate measurements of fission yields in neutron-induced fission, a proton-to-neutron converter (pnconverter) [6] and a dedicated ion guide [7] have been developed and tested at the IGISOL facility. However, the first test suggested that the production rate of exotic nuclei is lower than anticipated, hampering its usefulness in the intended studies. The production rate depends on several parameters, including the neutron flux from the pn-converter, the size and position of the actinide (uranium) targets, the volume and shape of the ion guide, and the helium gas pressure. To optimize the design with respect to the production of exotic 
nuclei, a simulation model based on GEANT4, GEF and MCNPX has been developed [8].

In this paper, we benchmark the simulation model against a measurement in which foils were placed at different positions in the ion guide [9]. Through $\gamma$-spectroscopy of these foils, the fission products stopped in the foils, as well as neutron activation products, were detected. The results of the measurements are compared to those expected from the model.

\section{Simulation model}

The simulation model combines MCNPX 2.5.0 [10], GEF 2020/1.1 [11] and GEANT4 10.2.3 [12]. MCNPX is used to simulate the production of neutrons from the beryllium pn-converter while the yields and kinetic energies of the fission products (FP) from neutron-induced fission of ${ }^{238} \mathrm{U}$ are obtained from GEF. The main part of the model is based on GEANT4 which is responsible for the transport of the fission products in the ion guide. The model and relevant details are described in previous publications $[8,13]$. The results presented in this paper have been obtained using GEANT4 version 10.2.3, where the transportation of the fission products was governed by the classes G4ionIonisation, G4hMultipleScattering (MS) and G4Nuclear Stopping [12].

Figure 1 shows the geometry of the GEANT4 model, which is based on the physical ion guide. The generation and transportation of nuclei (post neutron-emission fission products) in the model undergoes the following steps on an event-by-event basis:

1. A starting position is sampled uniformly in the uranium targets.

2. A neutron energy is sampled continuously and uniformly from 0 to $30 \mathrm{MeV}$ (the full energy of the proton beam).

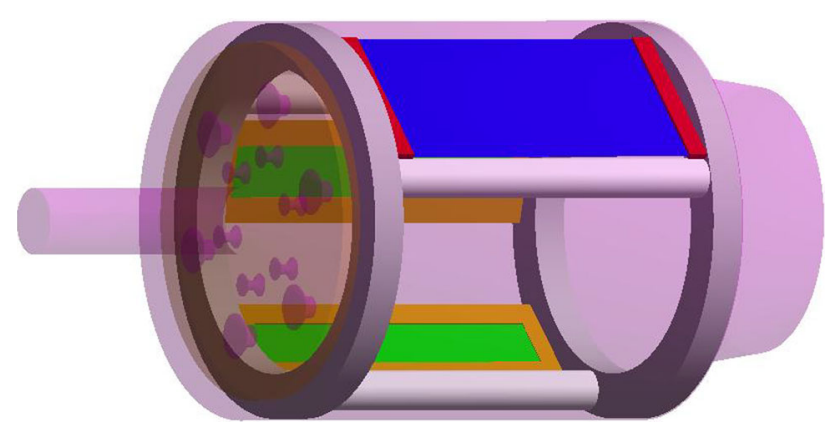

Fig. 1 Image of the geometry of the ion guide in the GEANT4 simulation. Purple: helium gas. Green: uranium targets. Blue: titanium foil. Orange and red: aluminium frames. Dark gray: aluminium supports. The outer walls are not shown to increase visibility
3. The mass (A), charge $(\mathrm{Z})$ and kinetic energy $\left(\mathrm{E}_{k i n}\right)$ of the nucleus is sampled from the GEF output of the corresponding neutron energy.

4. The nucleus is emitted isotropically, assuming negligible angular anisotropy and zero neutron momentum transfer, and then transported in the ion guide until fully stopped.

In one simulation, $10^{8}$ events (post neutron-emission fission products) are generated to ensure that the statistical uncertainty is negligible compared to systematic uncertainties, while keeping the computation time reasonable. To account for the distribution of the neutron flux in energy and space, the production rate of FPs is calculated by

$P=2 \times \sum^{N} \frac{\sigma\left(E_{n}\right) \times N_{U} \times \phi_{p}\left(E_{n}, \bar{r}\right) \times I_{p}}{N \cdot P\left(E_{n}\right)}$

where $\sigma\left(E_{n}\right)$ is the fission cross section at the neutron energy $E_{n}$, as obtained from the ENDF/B-VIII.0 evaluation [14] and $N_{U}$ is the number of uranium atoms in the targets. $\phi_{p}\left(E_{n}, \bar{r}\right)$ is the neutron fluence per proton at position $\bar{r}$ and neutron energy $E_{n}$, obtained from the MCNPX simulation, and $I_{p}$ is the proton intensity (protons per second) impinging on the pn-converter. $\mathrm{N}$ is the number of simulated events $\left(10^{8}\right)$ and $P\left(E_{n}\right)$ the probability of obtaining the neutron energy $E_{n}$ in the sampling process (Step 2). This energy is sampled uniformly from 30 energy bins between 0 and $30 \mathrm{MeV}$, and hence, $P\left(E_{n}\right)=\frac{1}{30}$. The factor 2 arises from the fact that two fission products are generated per fission event and assures that the total production rate of FPs is 2 times the fission rate. Each of the terms in Eq. (1), referring to a sampled position $\bar{r}$ and neutron energy $E_{n}$, is the multiplication factor used to calculate the yield of the corresponding FP event. Thus, by conditioning the production rate to a specific nucleus, $P(Z, A)$, the (absolute) independent fission yield (per second) of the nucleus is obtained. Furthermore, by also requiring the stopping position to be inside any of the foils the implantation rate of the chosen nucleus in that particular foil is obtained.

After the generation of the fission products, the stopping power of uranium and helium governs the collection. Because of the distribution of initial energy, position and direction of the FPs, some will lose enough energy to stop in the uranium targets while others will enter the helium gas or the aluminium backings. To investigate the energy dependence of the stopping range of uranium for different models, the stopping ranges of ${ }^{95} \mathrm{Zr}$ at energies from 40 to $100 \mathrm{MeV}$ were studied in natural uranium using TRIM [15] and different versions of GEANT4. A similar comparison was made for the helium gas for which the selected range of energies were 0.5 to $6 \mathrm{MeV}$.

In the previous version of the model [8], the GEANT4 class G4MultipleScattering, as well as the energy loss 
model G4IonParametrisedLossModel within the G4ionIonisation class, were not used. To investigate the performance of these models they were included in the study of stopping ranges and the results are presented in Fig. 2. As seen from Fig. 2, the G4MultipleScattering leads to a reduction in range and an increase in the spread of ranges of the stopped ${ }^{95} \mathrm{Zr}$ ions in both uranium and helium. Further more, including also the G4I on Parametrised Loss Model, the stopping ranges of ${ }^{95} \mathrm{Zr}$ in both uranium and helium increase and, in particular for the helium gas, get closer to the stopping ranges obtained with TRIM. The effect of the increases of the stopping ranges will be discussed together with the benchmark below.

\section{Measurement}

In the measurement of neutron-induced fission yields performed at the IGISOL facility [9], a proton beam with a nominal current of $10 \mu \mathrm{A}$ at an energy of $30 \mathrm{MeV}$ impinged on the $6 \mathrm{~mm}$ thick beryllium target of the pn-converter, and
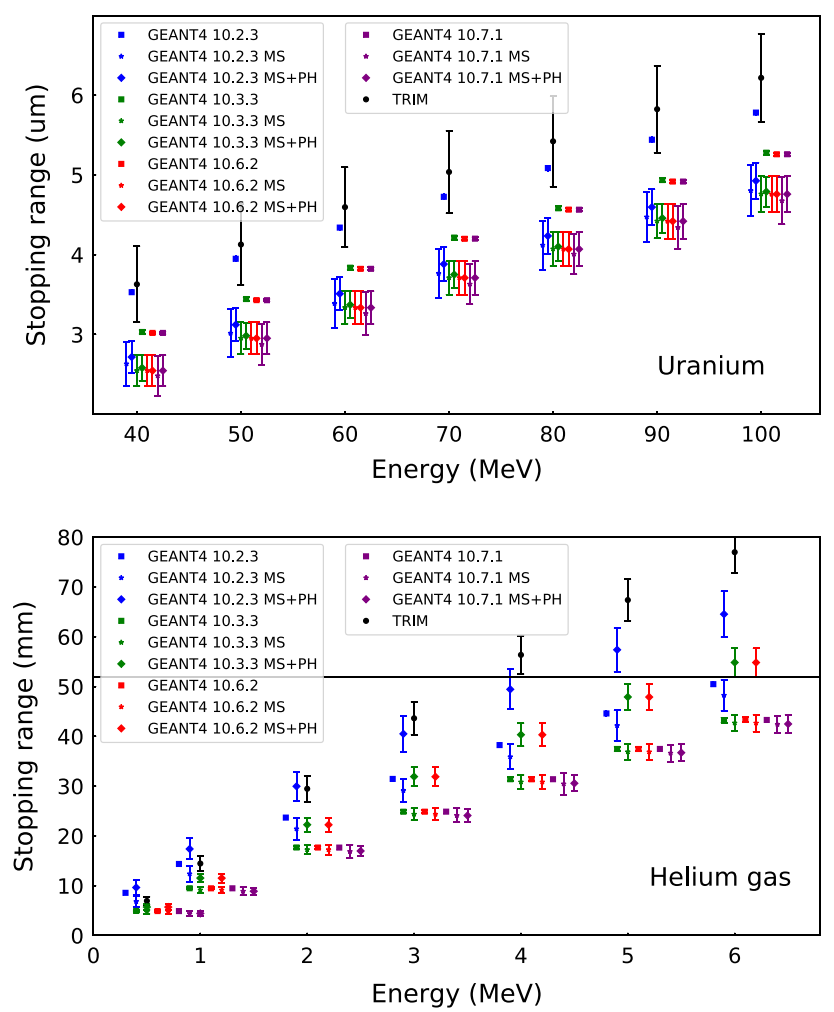

Fig. 2 Stopping ranges of ${ }^{95} \mathrm{Zr}$ in uranium (upper) and helium gas at a pressure of 400 mbar (lower) calculated with TRIM, GEANT4 10.2.3, 10.3.3, 10.6.2 and 10.7.1 with and without G4MultipleScattering (MS) and G4Ion Parametrised Loss Model (PH). The error bars represent standard deviations of the distributions of the stopping ranges instead of uncertainties. The horizontal line in the bottom panel represents the diameter of the ion guide the produced neutron flux induced fission in two uranium targets. The resulting fission fragments are either stopped in the uranium targets, the aluminium backings, in the helium gas or the titanium foil or hit the walls of the gas cell. At the same time, the neutrons also induced activation in the titanium foil and the uranium targets.

Figure 3 shows a schematic view of the setup implemented in the measurement. The two uranium targets, fixed to thick aluminium backings, were mounted in aluminium frames. The targets were made of natural uranium, each having a size of $10 \mathrm{~mm} \times 50 \mathrm{~mm}$ and thickness of $15 \mathrm{mg} / \mathrm{cm}^{2}$. However, $1 \mathrm{~mm}$ on each side was used to fix the target in the frames, resulting in an active area of each target of $8 \mathrm{~mm} \times 48 \mathrm{~mm}$ from which the FP enters the helium gas. On the opposite side of the uranium targets, a titanium foil with the size $24.5 \mathrm{~mm} \times 50 \mathrm{~mm}$ was installed. From the flow rate and the upstream pressure, the helium gas pressure in the ion guide was estimated to be 400(80) mbar.

After the beam was turned off, the uranium targets, the aluminium backings and the titanium foil were taken out of the ion guide. After a few days of cooling they were placed, one at a time, in front of a lead-shielded HPGe detector for measurements of $\gamma$ rays from fission and activation products.

As seen in Table 1, some of the foils were measured two or three times and the time evolution of the extracted counts were used to confirm the identifications by comparing to the expected decay and build-up of the corresponding nuclei. The dead times of the measurements are also listed in Table 1 and are adopted in the calculations of the intensities of the $\gamma$ rays.

The HPGe detector was energy calibrated with sources of ${ }^{60} \mathrm{Co},{ }^{133} \mathrm{Ba},{ }^{137} \mathrm{Cs}$ and ${ }^{241} \mathrm{Am}$. In addition to the energy calibration, an efficiency calibration, including geometrical efficiency, was made with sources of ${ }^{133} \mathrm{Ba},{ }^{152} \mathrm{Eu}$ and ${ }^{241} \mathrm{Am}$. The uncertainties from the calibrations are propagated in the analysis of the $\gamma$-spectroscopy data.

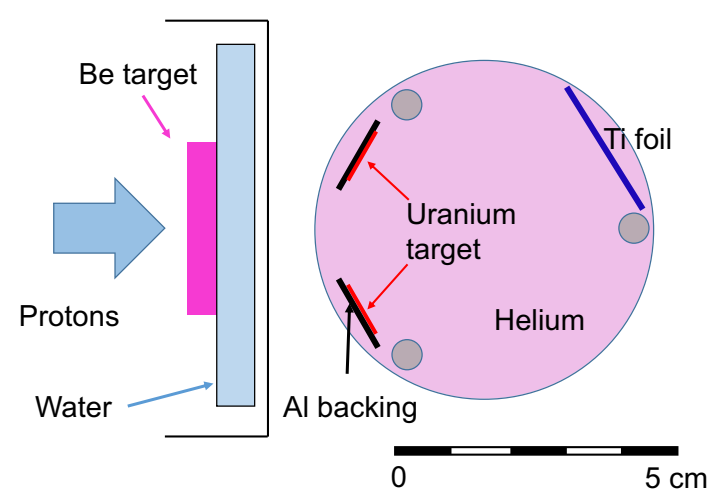

Fig. 3 A schematic view of the experimental setup 
Table 1 Measurements sequence for the different foils

\begin{tabular}{|c|c|c|c|c|}
\hline Measurement & $\begin{array}{l}\text { Start Time } \\
\text { mm:dd hh:mm }\end{array}$ & $\begin{array}{l}\text { Stop time } \\
\text { mm:dd hh:mm }\end{array}$ & $\begin{array}{l}\text { Duration } \\
\mathrm{h}\end{array}$ & $\begin{array}{l}\text { Dead time } \\
\%\end{array}$ \\
\hline Beam on & $12.0919: 40$ & $12.127: 00$ & 59.33 & \\
\hline Cooling down & $12.127: 00$ & $12.1712: 14$ & 125.23 & \\
\hline $\mathrm{U}$ target at bottom & $12.1712: 14$ & $12.1722: 04$ & 9.83 & 6.95 \\
\hline $\mathrm{U}$ target at top & $12.1722: 10$ & $12.187: 05$ & 8.92 & 6.48 \\
\hline Ti foil & $12.2010: 30$ & $12.2110: 33$ & 24.05 & 0.22 \\
\hline Both Al backings & $12.2113: 15$ & $12.2213: 21$ & 24.10 & 0.31 \\
\hline Al backings at top & $1.1911: 05$ & $1.2011: 08$ & 24.05 & 0.18 \\
\hline Al backing at bottom & $1.2011: 37$ & $1.2119: 41$ & 32.07 & 0.19 \\
\hline Ti foil & $1.2211: 23$ & $1.2311: 26$ & 24.05 & 0.18 \\
\hline Ti foil & $1.2316: 40$ & $1.248: 12$ & 15.53 & 0.17 \\
\hline
\end{tabular}

\section{Data analysis}

To benchmark the computer model using the spectroscopy data, the counts of identified $\gamma$-ray transitions extracted from the measurements and the simulations were compared.

\subsection{Identification of measured $\gamma$-ray transitions}

An example of the $\gamma$-spectrum from one of the uranium targets is presented in Fig. 4, with inserts of part of the spectra of the uranium target and the titanium foil. The energy and peak area for each $\gamma$-ray transition was extracted from such spectra of the different foils.

\subsubsection{Fission products implanted in the foils}

Some of the detected $\gamma$-ray transitions originate from the decays of fission products implanted in the foils. The implanted FP can be identified based on the measured $\gamma$ ray transitions. However, to confirm the presence of a FP in any of the foils, all transitions that are expected to have a detectable intensity, should be observed. In the initial analysis, $81 \gamma$-ray transitions in 12 decay chains were identified. However, only transitions that are unique to a certain FP, and could clearly be resolved from nearby peaks, were adopted in the benchmark. In addition, $\gamma$-ray intensities that could not be determined from at least two different foils are not useful

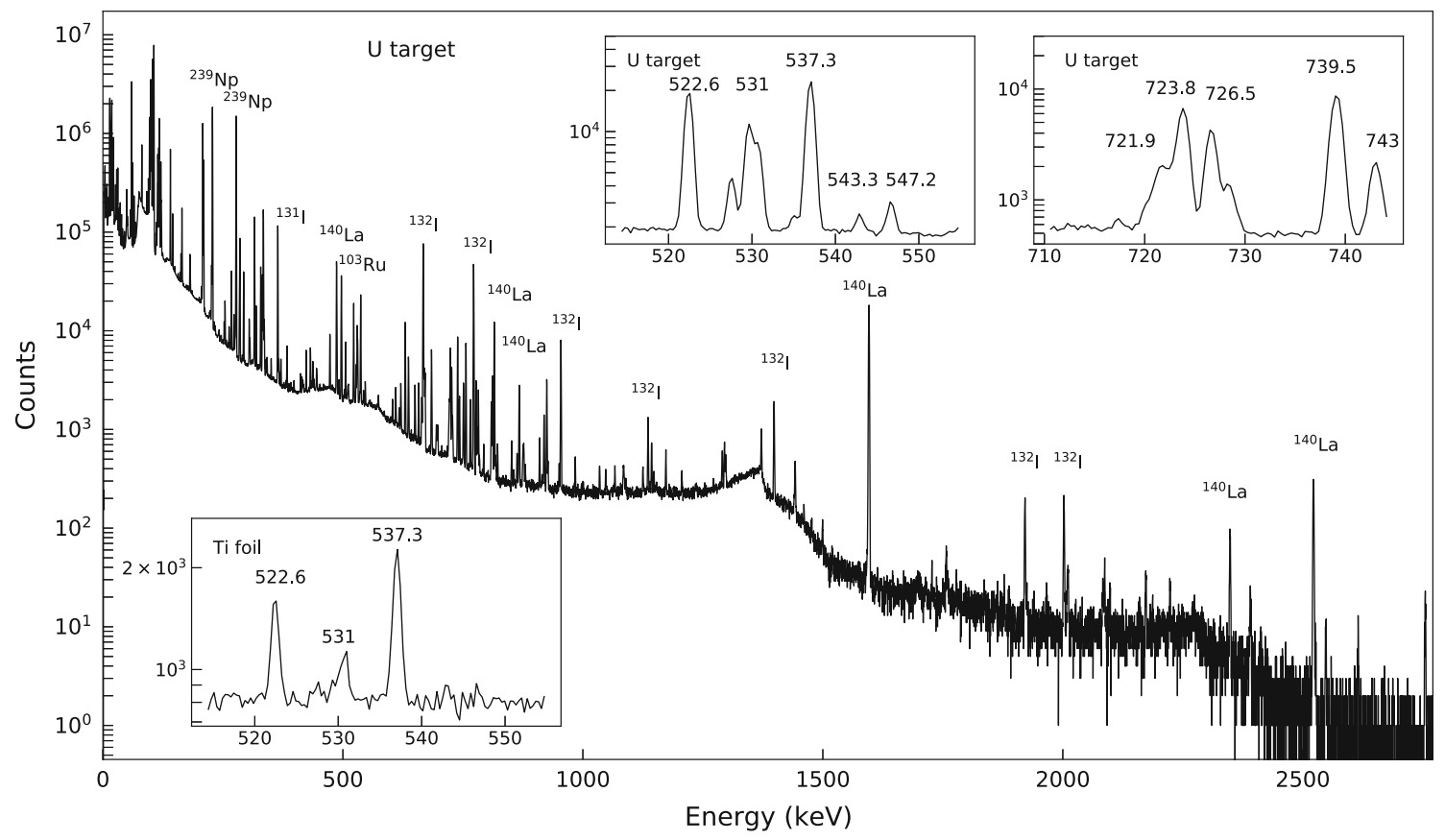

Fig. 4 Examples of $\gamma$-spectra obtained from the uranium target and the titanium foil 
Table 2 Observed $\gamma$-ray transitions from products of decay chains used in the benchmark

\begin{tabular}{|c|c|c|c|c|c|c|}
\hline $\begin{array}{l}\text { Energy } \\
(\mathrm{keV})\end{array}$ & $\begin{array}{l}\text { Branching } \\
\text { Ratio }\end{array}$ & $\begin{array}{l}\text { Parent } \\
\text { Nucleus }\end{array}$ & $R_{U}$ & $R_{A l}$ & $R_{T i}$ & Half-life \\
\hline 756.7 & $0.5438(22)$ & ${ }^{95} \mathrm{Zr}$ & $1.83(4)$ & $1.49(3)$ & $1.66(8)$ & $64.032 \mathrm{~d}$ [17] \\
\hline 765.8 & $0.99808(7)$ & ${ }^{95} \mathrm{Nb}$ & $1.77(5)$ & $1.47(3)$ & $1.51(7)$ & $34.991 \mathrm{~d}$ [17] \\
\hline 739.5 & 0.1220 & ${ }^{99} \mathrm{Mo}$ & $1.45(3)$ & $1.27(3)$ & $1.25(9)$ & $65.924 \mathrm{~h} \mathrm{[18]}$ \\
\hline 777.9 & $0.0431(8)$ & ${ }^{99} \mathrm{Mo}$ & $1.44(5)$ & $1.22(5)$ & $1.05(20)$ & \\
\hline 497.1 & $0.910(12)$ & ${ }^{103} \mathrm{Ru}$ & $1.62(4)$ & $1.35(3)$ & $1.36(4)$ & 39.247 d [19] \\
\hline 473.0 & $0.258(16)$ & ${ }^{127} \mathrm{Sb}$ & 1.14(8) & $1.04(8)$ & $0.84(12)$ & $3.85 \mathrm{~d}[20]$ \\
\hline 543.3 & $0.029(5)$ & ${ }^{127} \mathrm{Sb}$ & $1.18(27)$ & $1.04(22)$ & - & \\
\hline 685.7 & 0.368 & ${ }^{127} \mathrm{Sb}$ & $1.09(2)$ & $0.96(3)$ & $0.95(10)$ & \\
\hline 364.5 & $0.815(8)$ & ${ }^{131} \mathrm{I}^{a}$ & $1.31(3)$ & $1.17(3)$ & $1.09(3)$ & $8.0252 \mathrm{~d}[21]$ \\
\hline 637.0 & $0.0716(10)$ & ${ }^{131} \mathrm{I}$ & $1.34(4)$ & $1.18(4)$ & $1.16(12)$ & \\
\hline 262.9 & $0.0128(10)$ & ${ }^{132} \mathrm{I}^{a}$ & $1.25(12)$ & $1.02(11)$ & $0.88(48)$ & $2.295 \mathrm{~h} \mathrm{[22]}$ \\
\hline 505.8 & $0.0493(20)$ & ${ }^{132} \mathrm{I}$ & $1.51(8)$ & $1.57(10)$ & $1.40(19)$ & \\
\hline 522.6 & 0.16 & ${ }^{132} \mathrm{I}$ & $1.52(6)$ & $1.34(6)$ & $1.44(11)$ & \\
\hline 547.2 & $0.0114(8)$ & ${ }^{132} \mathrm{I}$ & $1.73(19)$ & $1.41(21)$ & - & \\
\hline *620.9 & $0.0197(28)$ & ${ }^{132} \mathrm{I}$ & $1.32(28)$ & $1.05(26)$ & - & \\
\hline 630.2 & $0.133(4)$ & ${ }^{132} \mathrm{I}$ & $1.41(5)$ & $1.31(6)$ & $1.27(11)$ & \\
\hline 650.5 & $0.0257(20)$ & ${ }^{132} \mathrm{I}$ & $1.43(13)$ & $1.45(19)$ & - & \\
\hline 667.7 & 0.987 & ${ }^{132} \mathrm{I}$ & $1.44(2)$ & $1.29(2)$ & $1.29(4)$ & \\
\hline 772.6 & $0.756(13)$ & ${ }^{132} \mathrm{I}$ & $1.42(3)$ & $1.34(4)$ & $1.22(5)$ & \\
\hline 954.6 & $0.176(15)$ & ${ }^{132} \mathrm{I}$ & $1.45(11)$ & $1.27(11)$ & $1.44(18)$ & \\
\hline 1136.0 & $0.031(14)$ & ${ }^{132} \mathrm{I}$ & $1.42(7)$ & $1.18(8)$ & - & \\
\hline 1143.3 & $0.0135(6)$ & ${ }^{132} \mathrm{I}$ & $1.37(10)$ & $1.14(11)$ & - & \\
\hline 1172.9 & $0.0109(7)$ & ${ }^{132} \mathrm{I}$ & $1.36(14)$ & $1.32(136)$ & - & \\
\hline 1290.8 & $0.0113(5)$ & ${ }^{132} \mathrm{I}$ & $1.24(10)$ & $1.24(16)$ & - & \\
\hline 1372.1 & $0.0247(10)$ & ${ }^{132} \mathrm{I}$ & $1.50(64)$ & $2.34(118)$ & - & \\
\hline 1398.6 & $0.0701(20)$ & ${ }^{132} \mathrm{I}$ & $1.43(7)$ & $1.30(8)$ & $1.27(13)$ & \\
\hline 1442.6 & $0.014(5)$ & ${ }^{132} \mathrm{I}$ & $1.24(9)$ & $1.23(15)$ & - & \\
\hline 1921.1 & $0.0123(6)$ & ${ }^{132} \mathrm{I}$ & $1.34(69)$ & $1.25(69)$ & $1.32(104)$ & \\
\hline 2002.2 & $0.0114(8)$ & ${ }^{132} \mathrm{I}$ & $1.20(13)$ & $1.08(14)$ & $1.37(41)$ & \\
\hline 304.8 & $.0429(6)$ & ${ }^{140} \mathrm{Ba}$ & $1.52(5)$ & $1.33(5)$ & $1.85(19)$ & $12.751 \mathrm{~d}[23]$ \\
\hline 423.7 & $0.0310(4)$ & ${ }^{140} \mathrm{Ba}$ & $1.60(6)$ & $1.52(6)$ & $1.21(15)$ & \\
\hline 537.3 & $0.2439(22)$ & ${ }^{140} \mathrm{Ba}$ & $2.00(5)$ & $1.75(5)$ & $1.51(7)$ & \\
\hline 328.8 & $0.203(3)$ & ${ }^{140} \mathrm{La}$ & $1.54(4)$ & $1.46(4)$ & $1.46(6)$ & $1.6786 \mathrm{~d}[23]$ \\
\hline 432.5 & $0.0290(3)$ & ${ }^{140} \mathrm{La}$ & $1.33(4)$ & $1.37(5)$ & $1.36(22)$ & \\
\hline 487.0 & $0.455(6)$ & ${ }^{140} \mathrm{La}$ & $1.73(4)$ & $1.52(4)$ & $1.63(6)$ & \\
\hline 751.6 & $0.0433(4)$ & ${ }^{140} \mathrm{La}$ & $1.60(5)$ & $1.42(4)$ & $2.20(65)$ & \\
\hline 815.7 & $0.2328(19)$ & ${ }^{140} \mathrm{La}$ & $1.57(3)$ & $1.44(3)$ & $1.61(8)$ & \\
\hline 867.8 & $0.0550(7)$ & ${ }^{140} \mathrm{La}$ & $1.66(4)$ & $1.59(5)$ & $1.46(17)$ & \\
\hline 919.6 & $0.0266(3)$ & ${ }^{140} \mathrm{La}$ & $1.52(4)$ & $1.41(5)$ & - & \\
\hline 925.2 & $0.0690(7)$ & ${ }^{140} \mathrm{La}$ & $1.52(4)$ & $1.37(4)$ & $2.21(36)$ & \\
\hline 1596.2 & $0.9540(8)$ & ${ }^{140} \mathrm{La}$ & $1.50(5)$ & $1.38(5)$ & $1.39(6)$ & \\
\hline 2521.4 & $0.0346(4)$ & ${ }^{140} \mathrm{La}$ & $1.56(11)$ & $1.39(13)$ & $1.30(20)$ & \\
\hline 145.4 & 0.484 & ${ }^{141} \mathrm{Ce}$ & $1.42(3)$ & $1.26(3)$ & $1.27(4)$ & $32.511 \mathrm{~d}[24]$ \\
\hline 293.3 & $0.428(4)$ & ${ }^{143} \mathrm{Ce}$ & $1.51(4)$ & $1.57(7)$ & $1.44(12)$ & $33.039 \mathrm{~h} \mathrm{[25]}$ \\
\hline
\end{tabular}


Table 2 continued

\begin{tabular}{lllllll}
\hline $\begin{array}{l}\text { Energy } \\
(\mathrm{keV})\end{array}$ & $\begin{array}{l}\text { Branching } \\
\text { Ratio }\end{array}$ & $\begin{array}{l}\text { Parent } \\
\text { Nucleus }\end{array}$ & $R_{U}$ & $R_{A l}$ & $R_{T i}$ & Half-life \\
\hline 350.6 & $0.0323(4)$ & ${ }^{143} \mathrm{Ce}$ & $1.62(8)$ & $1.24(27)$ & - \\
880.5 & $0.01031(13)$ & ${ }^{143} \mathrm{Ce}$ & $1.61(14)$ & - & - & $1.32(6)$ \\
91.1 & $0.281(7)$ & ${ }^{147} \mathrm{Nd}$ & $1.50(6)$ & $1.25(4)$ & $10.98 \mathrm{~d}[26]$ \\
\hline
\end{tabular}

The values in the columns $R_{U}, R_{A l}$, and $R_{T i}$ represent the comparisons of simulations to measurements of the different foils

*The $\gamma 620.9 \mathrm{keV}$ and $621.2 \mathrm{keV}$ are not resolvable from each other in the spectra. The tabulated branching ratios of them are summed up for one $\gamma$-ray transition

${ }^{a}$ The isomeric yield ratio of ${ }^{131} \mathrm{Te}$ and ${ }^{132} \mathrm{I}$ are estimated from the GEF simulation, and are adopted in the calculations below

for the consecutive analysis, and hence, were not utilised. Some details about the selection of the $\gamma$-ray transitions for the benchmark are presented in the examples below. All in all, $47 \gamma$-ray transitions from 10 decay chains remained for the comparisons. These transitions and their assignments are listed in Table 2. The values in the columns $R_{U}, R_{A l}$ and $R_{T i}$ represent the comparisons of simulations to measurements of the different foils, and will be described and discussed below.

As seen in the inserts of Fig. 4, the peak at $531 \mathrm{keV}$ was not resolvable from nearby peaks in the spectra of the uranium target and the titanium foil and hence, it was excluded from the comparison. The same is true for the peak at $723.8 \mathrm{keV}$ in the spectrum of the uranium target. Moreover there are also two sources for this peak, ${ }^{95} \mathrm{Zr}$ and ${ }^{131} \mathrm{I}$. Thus, the peak at $723.8 \mathrm{keV}$ could not be used for the benchmark, despite having a high intensity. On the other hand, although too weak to be observed in the spectrum of the titanium foil, the peaks at $543.3 \mathrm{keV}$ and $547.2 \mathrm{keV}$ could be resolved in the spectra of the uranium targets and the aluminium backings and hence, could be used in the benchmark.

Table 3 Observed $\gamma$-ray transitions from the products of neutron activation of the titanium foil and the uranium target used in the benchmark

\begin{tabular}{llll}
\hline $\begin{array}{l}\text { Energy } \\
(\mathrm{keV})\end{array}$ & $\begin{array}{l}\text { Branching } \\
\text { Ratio }\end{array}$ & $\begin{array}{l}\text { Parent } \\
\text { Nucleus }\end{array}$ & Reactions \\
\hline 889.3 & $0.999840(10)$ & ${ }^{46} \mathrm{Sc}[27]$ & ${ }^{46} \mathrm{Ti}(\mathrm{n}, \mathrm{p})$ \\
1120.5 & $0.999870(10)$ & & $\begin{array}{l}{ }^{47} \mathrm{Ti}(\mathrm{n}, \mathrm{d}) \\
{ }^{48} \mathrm{Ti}(\mathrm{n}, \mathrm{t})\end{array}$ \\
1297.1 & 0.67 & ${ }^{47} \mathrm{Ca} \mathrm{[28]}$ & ${ }^{50} \mathrm{Ti}(\mathrm{n}, \alpha)$ \\
175.4 & $0.0748(10)$ & ${ }^{48} \mathrm{Sc} \mathrm{[29]}$ & ${ }^{48} \mathrm{Ti}(\mathrm{n}, \mathrm{p})$ \\
1037.5 & $0.976(7)$ & & ${ }^{49} \mathrm{Ti}(\mathrm{n}, \mathrm{d})$ \\
1212.9 & $0.0238(4)$ & & ${ }^{50} \mathrm{Ti}(\mathrm{n}, \mathrm{t})$ \\
1312.1 & $1.001(7)$ & & \\
164.6 & $0.0186(3)$ & ${ }^{237} \mathrm{U} \mathrm{[30]}$ & ${ }^{238} \mathrm{U}(\mathrm{n}, 2 \mathrm{n})$ \\
\hline
\end{tabular}

\subsubsection{Neutron activation}

Some of the observed $\gamma$-ray transition in the titanium foil and the uranium targets originate from the decays of neutron activation products. From the intensities of these transitions the production rate of the activation products could be derived and used to benchmark the simulated neutron flux. As above, only unique and resolvable $\gamma$-ray transitions were used in the benchmark. These transitions, their branching ratios and the corresponding activation reactions are listed in Table 3.

\subsection{Calculations of $\gamma$-ray transitions from simulation}

\subsubsection{Fission products}

Using Eq. (1), with a condition that a specific FP stop in a specific foil, the corresponding implantation rate of the FP is obtained.

Starting from the implantation rates, considering the decay relationships of the corresponding decay chain, the build-up of each nuclide in the foils can be calculated. In these calculations, a time step length of $1 \mathrm{~min}$ is used. The implantation rate of any nuclide with a half-life shorter than one minute is added to the implantation rate of the daughter nuclide. The amount of a specific nuclide at the time step $\mathrm{m}, N^{m}$, is governed by

$N^{m}=N^{m-1}+D_{\text {pre }}^{m-1}-D^{m-1}+Y$

where $m$ is an index of the time step, $D^{m-1}=\lambda \times N^{m-1}$ is the number of decays during the time step $m-1, \lambda$ is the decay constant in units of per minute, and $\mathrm{Y}$ is the implantation rate in units of per minute. The subscript pre indicates the corresponding quantities of the decay precursor. Using Eq. (2), the amount of decays of a specific nuclide, during the measurement periods listed in Table 1, can be calculated for each measured foil. The expected counts of each $\gamma$-ray transition $\left(N_{\text {sim }}\right)$ are then calculated by multiplying with the tabulated branching ratios. These can then be compared with the observed counts, $N_{\text {exp }}$, of $\gamma$-ray transitions in the measurements. 
Figure 5 shows the $\mathrm{A}=95$ decay chain as an example. Since the precursors of ${ }^{95} \mathrm{Y}$ have shorter half-lives than 1 min, their implantation rates are added to the implantation rate of ${ }^{95} \mathrm{Y}$. Hence, according to the simulations, the implantation rate of ${ }^{95} \mathrm{Y}$ in the titanium foil is $3037.3 \mathrm{~s}^{-1}$ during irradiation. The corresponding implantation rate of ${ }^{95} \mathrm{Zr}$ is $7.4 \mathrm{~s}^{-1}$, while ${ }^{95} \mathrm{Nb}$ and ${ }^{95} \mathrm{Mo}$ have zero yields according to the GEF simulations. The decay chain is considered confirmed because two $\gamma$-ray transitions from the decay of ${ }^{95} \mathrm{Zr}$ and one from ${ }^{95} \mathrm{Nb}$ (marked with arrows in Fig. 5) were identified in the $\gamma$-spectra. The red arrows represent the $\gamma$-ray transition $756.7 \mathrm{keV}$ and $765.8 \mathrm{keV}$ that were used in the benchmark while as mentioned above the black arrow $(\gamma$ ray transition $724.2 \mathrm{keV}$ ) was excluded from the benchmark for having two sources, ${ }^{95} \mathrm{Zr}$ and ${ }^{131} \mathrm{I}$.

In Fig. 6, the simulated build-up of each isobar in the decay chain $\mathrm{A}=95$ is presented for the titanium foil. The vertical dotted line indicates when the beam was turned off. The dashed and shaded regions show the time-periods of the $\gamma$-ray spectroscopy measurements of the different foils (see Table 1). The black curve represents the total amount of nuclei of the decay chain.

\subsubsection{Calculations of neutron activation}

Table 3 lists the neutron activation products of titanium and uranium, which were identified by the observed $\gamma$-ray transitions. The production rates of these neutron activation products in the titanium foil and the uranium targets were calculated based on the simulated neutron flux from MCNPX and the cross sections from ENDF/B-VIII.0 [14] and JEFF-3.3 [16].

In Fig. 7, the neutron flux spectra from the MCNPX simulation and the calculated production rates of the neutron

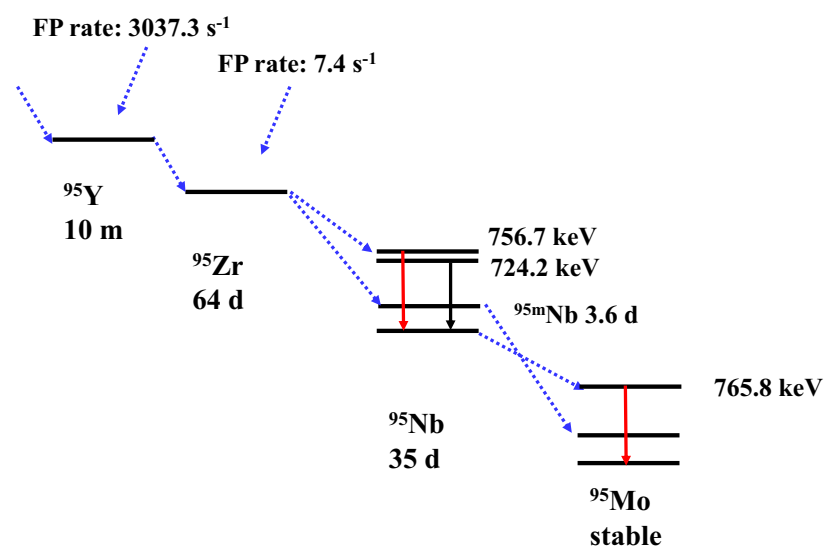

Fig. 5 Decay chain for mass number 95. The number after FP rate represents the corresponding implantation rate in the titanium foil extracted from the simulation, while the arrows are the observed transitions in the experimental data

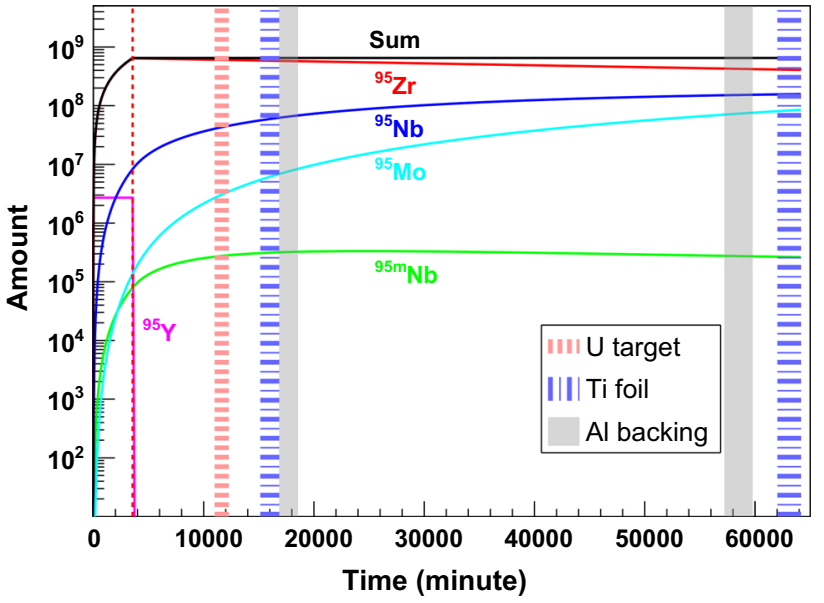

Fig. 6 Build-up of nuclides in the decay chain of $A=95$ based on simulated implantation rates. The vertical dotted line indicates when the beam was turned off. The dashed regions mark the time of the $\gamma$ measurements listed in Table 1

activation products in the titanium foil and the uranium target are presented. A build-up of the activation products was constructed in the same way as in the example shown in Sect. 4.2.1. The amount of decays of each activation product was calculated in the same way and then multiplied by the tabulated branching ratio of the transition. Hence, the expected counts of each transition $\left(N_{\text {sim }}\right)$ was obtained.

\subsection{Ratios of simulation to experiment}

The performance of the simulation model is evaluated by forming a ratio of each $\gamma$-ray transition

$R=\frac{N_{\text {sim }}}{N_{\text {exp }}}$

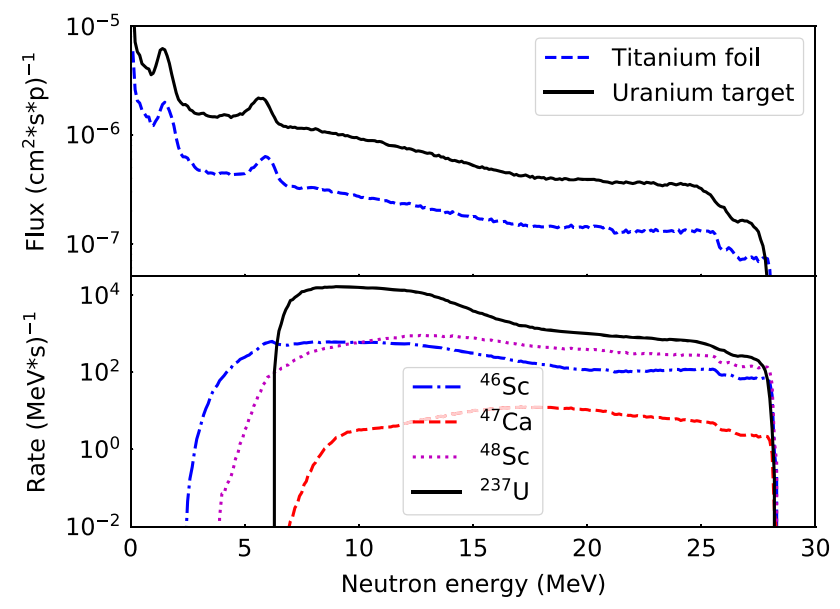

Fig. 7 The neutron flux spectra from MCNPX and the calculated production rates of the neutron activation products in the titanium foil and the uranium target 
where $N_{\text {sim }}$ is the calculated number of $\gamma$-rays from the simulation and $N_{\text {exp }}$ is the number of counts extracted from the spectroscopy data.

In the estimation of the uncertainty of the calculated number of $\gamma$-rays from the simulation $\left(\delta N_{\text {sim }}\right)$ only the uncertainties of tabulated branching ratios are included. The uncertainties of the half-lifes can be ignored because their impact is very small. Also the effect of $\beta$-delayed neutron emission has been estimated and deemed insignificant. The uncertainties of the FY from GEF are not included at this stage, but should be considered in future work. Considering that $1 \times 10^{8}$ fissions per neutron energy are calculated by GEF, and that each run of the GEANT4-simulation includes the same number of events, the statistical uncertainties from the simulations are negligible compared to uncertainties from other sources.

The measured number of counts is derived from the peak area, corrected by the corresponding efficiency. Thus, the uncertainty of the measured counts $\left(\delta N_{\text {exp }}\right)$ have contributions from the uncertainties of the peak areas and the efficiency calibration. For all $\gamma$-ray transitions that were measured more than once in a certain foil (see Table 1) the uncertainties of the peak areas extracted from the measurements were treated as statistical uncertainties and were propagated in the calculation of the weighted average ratio for that $\gamma$-ray, while the uncertainties from the efficiency calibration were added after.

Hence, the uncertainties, $\delta R$, of the ratios listed in Table 2 include the uncertainties of the branching ratios, the peak areas, and the efficiency calibration.

In the subsequent analysis, average ratios from different foils (or decay chains) are compared in order to draw conclusions about the accuracy of the simulation model. These averages were formed using the estimated uncertainties as weights, $w=\frac{1}{\delta R^{2}}$. In the estimation of the uncertainties of the average ratios, the uncertainties $\delta R$ are assumed to be independent. The estimates of the uncertainties were derived in two different ways: using error propagation

$\delta R_{\text {prop }}=\frac{1}{\sqrt{\sum w_{i}}}$,

and using the weighted standard error of the mean

$\delta R_{s t d}=\sqrt{\frac{\sum w_{i}\left(R_{i}-R\right)^{2}}{(N-1) \sum w_{i}}}$.

The index $i$ refers to the different $\gamma$-ray transitions from a certain mass number or foil. Assuming large sample sizes and no unknown uncertainties these two measures should be identical. In cases where the two estimates disagree the larger of the two is adopted as the uncertainty of the average ratio.

\section{Results and discussion}

As mentioned above, the products of neutron activation and the fission products were identified from the foils. The ratios from the activation data can be used to benchmark the neutron production from the MCNPX simulation. On the other side, comparisons of $R$-values based on implantation data from the different foils are used to benchmark the transportation of fission products in the GEANT4 simulation. For example, a comparison of the average ratio from the aluminium backings $\left(R_{A l}\right)$ with that from the titanium foil $\left(R_{T i}\right)$ can be used to benchmark the ion transport in the helium gas, i.e. the stopping efficiency of the gas.

\subsection{Neutron flux}

The production rates of the observed activation products in the titanium foils and the uranium targets are dominated by high-energy neutrons (see Fig. 7). Hence, the ratios of simulations to measurements of the activation products can be used to benchmark the high energy part of the neutron flux obtained from the MCNPX simulation. Figure 8 shows the ratios from the observed activation products together with the weighted average ratio $1.42(4)$.

It is noticeable that the ratio at $175.4 \mathrm{keV}$ deviates significantly from those from the same nuclide $\left({ }^{48} \mathrm{Sc}\right)$ at higher energies. However, whereas this deviation can not be really understood, disregarding the ${ }^{48} \mathrm{Sc}$ results from the benchmark does not significantly change the average ratio.

The fact that the activation ratio is significantly larger than one implies that the flux of high-energy neutrons in the current simulation is overestimated. This conclusion is supported by similar overestimations of fission products stopped in the foils (see Sect. 5.2).

The reason for the overestimation of the neutron flux is not known but the relatively good agreement between the activation of the uranium targets and the titanium foil (see Fig. 8) indicates that the geometry of the simulation model is in reasonable agreement with the experimental setup. Possible explanations for the overestimation include the estimation of the integrated beam current on the target, and the modeling of the neutron production in the MCNPX simulation.

In the measurement, the proton beam current could not be monitored online. Instead it was measured from time to time by blocking the beam with a non-penetrable Faraday cup in front of the reaction chamber. In addition, neither the beam size nor the beam position on target could be measured. Hence, the integrated beam current on target, used in the calculation of the $\gamma$-intensities from the simulation, had to be inferred from the readings of the Faraday cup. In future versions of the neutron converter, an online monitoring of the proton beam current on the beryllium target is anticipated. 


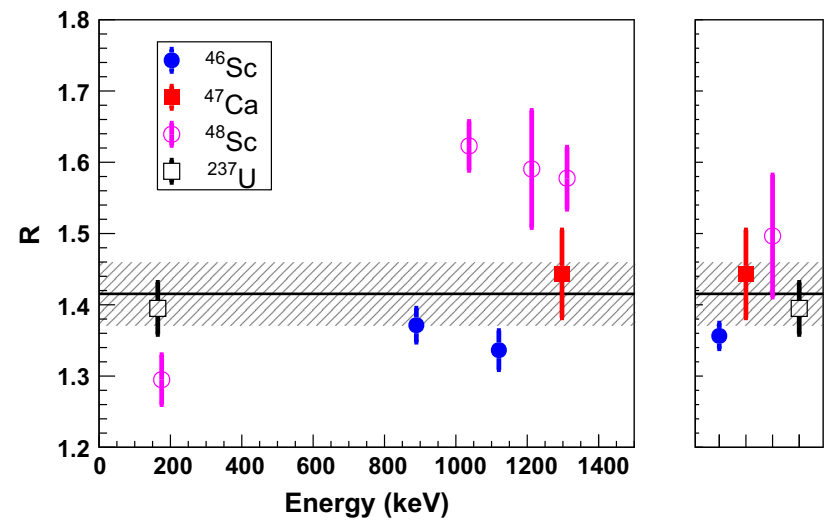

Fig. 8 Left panel: $R$-values for $\gamma$-ray transitions from the activation of the titanium foil and the uranium targets as a function of energy. Right panel: average $R$-value of each activation product. The horizontal line and the dashed region represent the overall weighted average ratio 1.42 with an uncertainty of 0.04

The $30 \mathrm{MeV}$ proton beam is fully stopped in the $6 \mathrm{~mm}$ thick Beryllium target. This is taken into account in the MCNPX-simulation of the neutron production where the interaction of the proton beam with the beryllium target is governed by the endf70prot library [31]. However, the resulting neutron flux in the ion guide will depend on the double differential neutron production cross sections of multiple neutron producing channels $(\mathrm{Be}(\mathrm{p}, \mathrm{xn}))$ at proton energies ranging from 0 to $30 \mathrm{MeV}$. To what degree this can be accurately modeled by MCNPX is beyond the scope of this study. However, it is not unreasonable to assume that at least part of the $40 \%$ deviation between simulated and experimental results could be explained by model defects.

It is also worth mentioning that ${ }^{239} \mathrm{~Np}$, a daughter of the neutron activation product ${ }^{239} \mathrm{U}$, was observed in the spectrum from the uranium targets. The reaction ${ }^{238} \mathrm{U}(\mathrm{n}, \gamma)$ has a much higher cross section at low-energy than at high-energy [14]. Thus, the ratios derived from ${ }^{239} \mathrm{~Np}$ can be seen as an indicator for the fluence of low-energy neutrons. The $R$ values of the $\gamma$-ray transitions $106.1 \mathrm{keV}$ and $277.6 \mathrm{keV}$ belonging to the nucleus ${ }^{239} \mathrm{~Np}$, are $0.090(2)$ and $0.094(2)$ respectively. The weighted average of these, 0.092(2), is about 10 times smaller than one, suggesting that the neutron fluence at low energies, as obtained from the current MCNPX simulation, is underestimated.

The neutron field produced by the neutron converter is a white spectrum ranging from thermal energies to $30 \mathrm{MeV}$. However, in the simulation model the response from the surroundings has been ignored, which could explain the underestimation of the low energy flux. As a result, more than $98 \%$ of the fissions in the simulation originate from ${ }^{238} U(n, f)$. Considering that the fission rate of ${ }^{235} \mathrm{U}(\mathrm{n}, \mathrm{f})$ is dominated by low-energy neutrons it is likely that the fission rate of ${ }^{235} \mathrm{U}(\mathrm{n}, \mathrm{f})$ is also heavily underestimated. A more accurate model of the neutron flux would include the response of the surrounding environment, which might add a non-negligible contribution from thermal fission of ${ }^{235} \mathrm{U}$.

\subsection{Transportation and stopping of fission products}

The $R$-values for each observed $\gamma$-ray transition belonging to FPs in the foils are listed in Table 2. From these values, the weighted average ratios of $\gamma$-ray transitions from the same mass chain are calculated per foil and shown in Fig. 9. The grand average ratios with respect to the foils are also calculated, $R_{U}=1.47$ (3) for the uranium targets, $R_{A l}=1.33$ (2) for the aluminium backings, and $R_{T i}=1.31$ (3) for the titanium foil, respectively. These ratios all indicate that the production of FPs in the simulation is overestimated, which is consistent with the overestimation of neutron activation discussed above. Keeping this overall overestimation in mind, the differences between the average ratios of the different foils are discussed below.

A weighted two-sample t-test shows that the ratio from the uranium targets are larger than that from the aluminium backings at a $99 \%$ significance level. The difference means that more FPs remain in the uranium targets and less reach the aluminium backings in the simulation compared to the experimental result. According to the simulation, $70.9 \%$ of the generated fission fragments stop in the uranium targets and $14.7 \%$ in the aluminium backings (see Table 4). From this, the size of the overestimation of the stopping in the uranium can be estimated to be $3 \%$. Likewise, an underestimation of the stopping in the aluminium of about $7 \%$ is obtained. Many factors, including; the shape, uniformity and thickness of the targets; the angular anisotropy of the FPs;

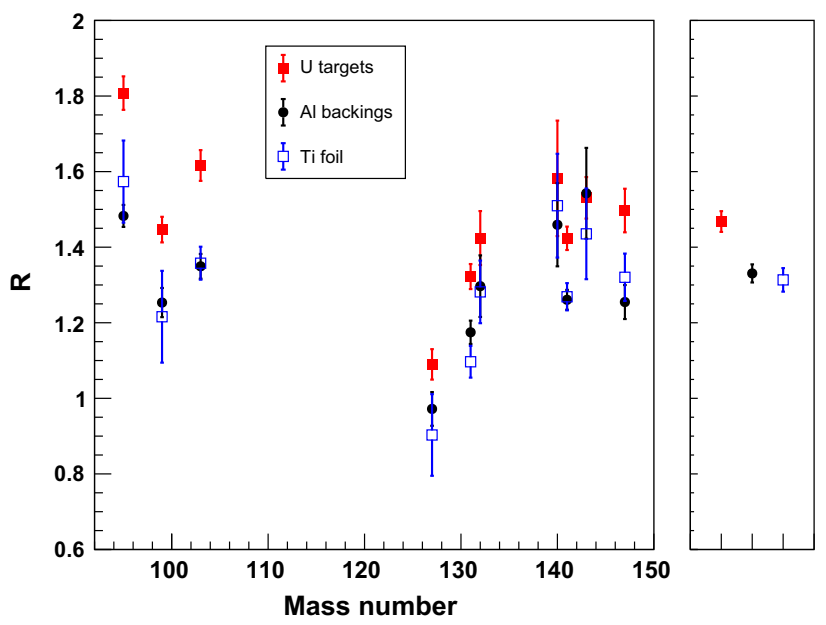

Fig. 9 The weighted average $R$ for each mass chain obtained from the uranium targets, the aluminium-backings and the titanium foil. The right panel shows the weighted average ratios with uncertainties for the different foils 
Table 4 The fraction of fission products stopped in different parts of the ion guide, together with average ratios of simulation to experiment from the foils

\begin{tabular}{|c|c|c|c|c|}
\hline & $\mathrm{V}-10.2 .3$ & V-10.3.3 & V-10.6.2 & V-10.7.1 \\
\hline Stopping in the uranium targets & $70.9 \%$ & $72.6 \%$ & $72.6 \%$ & $72.5 \%$ \\
\hline Stopping in the aluminium backings & $14.7 \%$ & $13.9 \%$ & $13.8 \%$ & $13.8 \%$ \\
\hline Stopping in the helium gas & $0.87 \%$ & $1.09 \%$ & $1.09 \%$ & $1.60 \%$ \\
\hline Stopping in the titanium foil & $1.03 \%$ & $0.95 \%$ & $0.95 \%$ & $0.88 \%$ \\
\hline $\mathrm{R}_{U}$ & $1.47(3)$ & $1.51(3)$ & $1.50(3)$ & $1.51(3)$ \\
\hline $\mathrm{R}_{A l}$ & $1.33(2)$ & $1.24(2)$ & $1.24(2)$ & $1.24(2)$ \\
\hline $\mathbf{R}_{T i}$ & $1.31(3)$ & $1.16(3)$ & $1.18(3)$ & 1.09 (3) \\
\hline
\end{tabular}

The data is presented for simulations using different versions of GEANT4

the neutron momentum transfer; and the stopping power of uranium, could contribute to this discrepancy.

Depending on the starting positions in the target, the fission products that leave the target will have energies ranging from zero up to the full energy of the initial fragments (of the order of $100 \mathrm{MeV}$ ). After entering the helium gas the slowing down of the FPs continues. If the remaining kinetic energy of a FP is completely absorbed by the helium gas, the FP will be stopped in the gas and transported out of the ion guide by the gas flow. If the FP passes through the helium gas it will either hit the walls of the ion guide or stop in the titanium foil. Table 4 lists the fractions of simulated FPs stopped in different parts of the ion guide for different versions of GEANT4.

There is no material between the uranium targets and the aluminium backings, while there is the helium gas between the targets and the titanium foil. Thus, a comparison between the ratios of fission products stopped in the aluminium backings and the titanium foil can be used to benchmark the stopping performance of the helium gas. The ratios from the aluminium backings and the titanium foil agree within their respective uncertainties, and a t-test shows the difference to be insignificant. Hence, we conclude that the stopping efficiency of the helium gas obtained from the simulation agrees with the experimental results.

The model of the ion guide presented in this paper was developed based on version 10.2.3 of GEANT4 with the class G4MultipleScattering (MS) and the energy loss model G4Ion ParametrisedLoss Model (PH). To investigate the impacts of recent updates of the program the simulations were repeated using different versions of GEANT4 and the results are presented in Table 4. This shows that version 10.2.3 yields less FPs stopped in the uranium and more in the aluminium compared to the newer versions. Version 10.2.3 also give less FPs stopped in the helium gas compared to the other versions. Both of these results are consistent with the calculated stopping ranges of ${ }^{95} \mathrm{Zr}$ obtained with the different versions of GEANT4 (see Fig. 2).

According to the $R$-values of the foils shown in Table 4, GEANT4 10.2.3 agrees best with the measurements. In Table 4, the values from the simulation with GEANT4 10.2.3 are closest to the average ratio from the neutron activation data of 1.42. Furthermore, the deviation between $R_{U}$ and $R_{A l}$ for the simulation with version 10.2.3 are smaller than that for the simulations by other versions of GEANT4. Also, $R_{A l}$ agrees with $R_{T i}$ within uncertainty for the simulation by GEANT4 10.2.3 but not for the others. Hence, we conclude that GEANT4 10.2.3 results in the best agreement between simulation and experiment.

The implementation of the class G4MultipleScattering (MS) and the energy loss model G4Ion Parametrised Loss Model (PH) has an impact on the $R$-values consistent with the change of stopping ranges shown in Fig. 2. Omitting them from the simulation results in much worse agreement between simulation and experiment. Thus, we conclude that both of these play an important role in current simulation model and should be adopted in future models.

According to the simulation by GEANT4 10.2.3, less than $1 \%$ of the produced fission products are stopped in the helium gas.

\section{Conclusions and outlook}

In this work we have used three independent Monte Carlo codes; GEF, GEANT4 and MCNPX, with the aim of modelling the generation and collection of fission products in the IGISOL neutron-induced fission ion guide. The MCNPX calculation of the production of neutrons in the proton-to-neutron converter depends on the $\mathrm{Be}(\mathrm{p}, \mathrm{xn})$ doubledifferential cross section. Due to energy absorption of the protons in the beryllium target [6], this cross section has to be known as a function of proton energy. The neutron production heavily depends on the proton beam current and, to a lesser extent, the size of the beam spot. With this in mind, the observed overestimation of the neutron flux by approximately $40 \%$ does not seem unreasonable.

In the second step of the simulation, GEF is used to generate fission products from the ${ }^{238} \mathrm{U}(\mathrm{n}, \mathrm{f})$ reaction at a neutron energy of 0 to $30 \mathrm{MeV}$. However, the reliability of GEF depends on the availability of experimental data and, espe- 
cially at the higher part of the neutron energy spectrum and for fission products with low yields, data are scarce. This is a possible explanation for what seems to be a significant and systematic decrease of the ratios for mass chain 127 and 131 in Fig. 9.

For this study the performance of different versions of GEANT4 (10.2.3, 10.3.3, 10.6.2, and 10.7.1) were investigated. Although similar results were obtained in all cases, the version which best reproduces the data is 10.2.3, resulting in an agreement between the simulations and the experiment well within $10 \%$.

Considering the many steps involved, from the incoming proton beam until a fission product is stopped, and the fact that three different Monte Carlo codes have been used, the predictive power of the model is very good and sufficient for the purpose of modelling the ion guide.

The next step will be to use the model to optimize the design of a new ion guide to increase the amount of extracted fission products. In the measurement of neutron-induced fission yields [9] the amount of fission products extracted from the ion guide was too low to perform the intended measurement. To improve this the geometry could be modified to allow an even shorter distance between neutron production target and fission target. The design could also be optimized to increase the collection efficiency of the helium gas. Due to vacuum requirements downstream, a higher helium pressure is not a feasible way to achieve this and instead the volume of the ion guide has to be increased. This in turn will probably require a RF-structure that provide an electric field guidance system, similar to the CARIBU gas catcher [32], to achieve a sufficiently efficient collection and extraction of the fission products from the guide. Such a RF-structure will be adjusted to the size of the IGISOL ion guide and tested with a Californium source before it is put in use in experiment.

Acknowledgements We thank K. Jansson for developing the initial version of the GEANT4 model. This work was supported by the Swedish research council Vetenskapsrådet (Ref. No. 2017-06481), the European Commission within the Seventh Framework Programme through Fission-2013-CHANDA (Project No. 605203), the Swedish Radiation Safety Authority (SSM), and the Swedish Nuclear Fuel and Waste Management Co. (SKB).

Funding Information Open access funding provided by Uppsala University.

Data Availability Statement This manuscript has no associated data or the data will not be deposited. [Authors' comment: The data is stored in the database of the research group and can be accessed to by contacting the corresponding author.]

Open Access This article is licensed under a Creative Commons Attribution 4.0 International License, which permits use, sharing, adaptation, distribution and reproduction in any medium or format, as long as you give appropriate credit to the original author(s) and the source, provide a link to the Creative Commons licence, and indicate if changes were made. The images or other third party material in this article are included in the article's Creative Commons licence, unless indicated otherwise in a credit line to the material. If material is not included in the article's Creative Commons licence and your intended use is not permitted by statutory regulation or exceeds the permitted use, you will need to obtain permission directly from the copyright holder. To view a copy of this licence, visit http://creativecomm ons.org/licenses/by/4.0/.

\section{References}

1. I.D. Moore, P. Dendooven, J. Ärje, Hyperfine Interact 223, 17 (2014)

2. G. Lhersonneau et al., Eur. Phys. J. A 9, 385 (2000)

3. H. Penttilä et al., Eur. Phys. J. A 48, 143 (2012)

4. V. Rakopoulos et al., Phys. Rev. C 98, 024612 (2018)

5. V. Rakopoulos et al., Phys. Rev. C 99, 014617 (2019)

6. A. Mattera et al., Eur. Phys. J. A 53, 173 (2017)

7. D. Gorelov et al., Nucl. Instrum. Methods B 376, 46 (2016)

8. K. Jansson et al., Eur. Phys. J. A 53, 243 (2017)

9. A. Mattera et al., Eur. Phys. J. A 54, 33 (2018)

10. G.W. McKinney et al., MCNPX 2.5.0—new features demonstrated, in Proceedings of the MC2005 conference, Chattanooga, Tennessee, April 17-21, 2005 (American Nuclear Society, 2005) LAUR-04-8695

11. K.H. Schmidt et al., Nucl. Data Sheets 131, 107 (2016)

12. S. Agostinelli et al., Nucl. Instrum. Methods A 506, 250 (2003)

13. A. Al-Adili et al., Eur. Phys. J. A 51, 59 (2015)

14. D.A. Brown et al., Nucl. Data Sheets 148, 1 (2018)

15. J. Ziegler, J. Biersack, U. Littmark, The Stopping and Ranges of Ions in Solids, Chapter 1 (Pergamon, Oxford, 1985)

16. A.J. Koning, D. Rochman, Nucl. Data Sheets 113, 2841 (2012)

17. S.K. Basu, G. Mukherjee, A.A. Sonzogni, Nucl. Data Sheets 111, $2555(2010)$

18. E. Browne, J.K. Tuli, Nucl. Data Sheets 145, 25 (2017)

19. D. De Frenne, Nucl. Data Sheets 110, 2081 (2009)

20. A. Hashizume, Nucl. Data Sheets 112, 1647 (2011)

21. Yu. Khazov, I. Mitropolsky, A. Rodionov, Nucl. Data Sheets 107, 2715 (2006)

22. Yu. Khazov, A.A. Rodionov, S. Sakharov, B. Singh, Nucl. Data Sheets 104, 497 (2005)

23. N. Nica, Nucl. Data Sheets 154, 1 (2018)

24. N. Nica, Nucl. Data Sheets 122, 1 (2014)

25. E. Browne, J.K. Tuli, Nucl. Data Sheets 113, 715 (2012)

26. N. Nica, Nucl. Data Sheets 110, 749 (2009)

27. S.-C. Wu, Nucl. Data Sheets 91, 1 (2000)

28. T.W. Burrows, Nucl. Data Sheets 108, 923 (2007)

29. T.W. Burrows, Nucl. Data Sheets 107, 1747 (2006)

30. M.S. Basunia, Nucl. Data Sheets 107, 3323 (2006)

31. R.C. Little et al., ENDF70: A Continuous-Energy Neutron Data Library Based on ENDF/B-VII.0, LA-UR-08-2909 (2008)

32. G. Savard et al., Nucl. Instrum. Methods B 376, 246 (2016) 